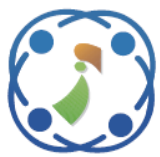

\title{
Human Motion Detection through Wall Based on Micro-Doppler Using Kalman Filter Combined with Convolutional Neural Network
}

\author{
Thamir R. Saeed ${ }^{1 *}$ \\ Saad Mutashar $^{1} \quad$ Aqeela N. Abed ${ }^{2}$ \\ Mahmuod Hamza Al-Muifraje ${ }^{1}$ \\ Ahlam Luaibi Shuraiji ${ }^{3} \quad$ Shaymaa Abd-Elghany $^{1}$ \\ ${ }^{1}$ Electrical Engineering Department, University of Technology, Iraq \\ ${ }^{2}$ Faculty of Medicine, University of Iraq, Iraq \\ ${ }^{3}$ Electromechanical Engineering Department, University of Technology, Iraq \\ * Corresponding author’s Email: thamir_rashed@yahoo.com
}

\begin{abstract}
Human motion detection, classification, and perceiving, the dynamics of moving objects in the environment, are crucial in many applications. Many sensors have been used to this detection; the radar represents one of the promising sensors. Kalman filter $(\mathrm{KF})$ and convolutional neural network $(\mathrm{CNN})$ represents a powerful tool for estimation and classification respectively. In this paper, a combination between the KF and CNN have been proposed to detect and classify human behavior. This proposal presents two important points, the precise features map from the combination of Kalman Filter and CNN, as well as the use of the radar, which is working under all circumstances and does not break the privacy. Twenty different experiments with three scenarios for different motion with and without glass wall have been studied, and they are classified. The results show that the overperform of the proposed algorithm and the classification accuracy can reach 98.7\%. This advancement of the proposed algorithm depends on the efficient Wigner-Ville short time Fourier transform (STFT) which is used as a feature extractor and make Range-Doppler (RD) map.
\end{abstract}

Keywords: Kalman filter, CNN, Human motion recognition, STFT.

\section{Introduction}

Human behavioral monitoring by a conventional monitoring sensor with or without a barrier is considered interesting topics. The monitoring process depends on the monitoring sensors and monitoring areas. There are many types of monitoring sensors like a video camera, laser, infrared and radar. Each one of these sensors has a specific limitation. The monitoring areas such as; suspicious event, sporadic conduct, uncommon behavior, unusual behavior, abnormal behavior, and anomaly [1-4].

The most common sensors in this area are cameras, but there are many weak points when using the camera for monitoring; the blind spot is generated, and cannot use it in everywhere and at all time [5]. As well as, when tracking an object which has the same color with other objects or with the background, the tracking process will be affected negatively [6]. However, all of the vision-based sensors suffer limitations in different lighting and weather conditions.

Therefore, the challenge in this subject is to provide efficient monitoring sensor under different environment without breaking the privacy. However, the detection process remains complex, so intelligent algorithms have been used to detect, classify and then recognize certain behaviors and activities [7, 8]. In this context, radar sensors present advantages for human motion detection and identification because of their working in allweather and all-time capabilities, besides the fact that they detect targets at a long range, and behind obstacles [9-11].

The features of each moving object represent the signatures for classifying and recognize these 
objects. Many researchers explore various features (representation) learning methods for highresolution object recognition. Therefore, in modern radar technology, especially, the high-resolution radars in the short-range are extracting the microDoppler (MD) as a feature for detection, recognition and tracking the human motion [12, 13]. In this context, each moving object has a unique MD and micro-Range (MR) signature that can be used for classification of this object. [2].

Therefore, in the detection and recognition of human motion, many existing works are presenting different techniques and sensors for human detection and activity recognition, which are different from our work. In [2] a $25 \mathrm{GHz}$ radar is used, and MD signatures are used with conventional machine learning and Deep Convolutional Neural Networks (DCNN) approach to detect possible human presence. In [9] was used Method of Moments enhanced with Fast Multipole Method (MoM-FMM) with $5 \mathrm{GHz}$ radar, this algorithm is used for scattering model. Therefore, it depends on the scattering energy, and the efficient classification can be gained for the direct motion only. The author in [14] focused on distinguishing the human target from different objects based on their corresponding MD signatures. Others focused on using radar for safe pedestrian recognition in the automotive industry to apply an emergency brake to the car when a pedestrian is detected at a close distance [15]. Belgiovane et al. [16], use the camera of walking and running pedestrian to input an MD simulation. Afterward, measured MD signals of a radially and laterally walking pedestrian in the $77 \mathrm{GHz}$ radar. In [17], attempts to decompose human MD signals are presented. The movement behavior of a marked subject is recorded by infrared cameras. Then for simplicity and comparison, simulate the radar return signal at in the $24 \mathrm{GHz}$ band, to measure the MD for human motion. Reference [18], present an approach to extend the standard time-frequency representation of MD signals by the distance dimension. Where [4] present an additional consideration of the range dimension to separate body parts when using highresolution radars. In [19] a body part Range-velocity (Doppler) feature map is considered, and a tree classifier is used. Decision Tree Classifier repetitively divides the working area into subpart by identifying thresholds.

The impurity of this algorithm is when having traces of one class division into others, therefore the classification rate is low. In [20] was used as an electroencephalogram (EEG) and galvanic skin response (GSR) signals to emotion recognition. A zero-crossing rate is used for pre-processing the
GSR single. While sufficient EEG feature extraction was obtained through $\mathrm{CNN}$. He uses $\mathrm{CNN}$ for improving the recognition rate, while the reduction of that rate is in the EEG part by using zero-crossing processing. In [21] was used a body mass index for indicating the body's height-weight, while a conventional autoencoder (CAE) is used to extract the latent features from the images. A further dimension reduction is applied using the $t$ distributed stochastic neighbor embedding (t-SNE). A good identifying of the micro-Doppler signature with high SNR was gained by $98 \%$. The gap of the previous work is concentrated in two points; the combination algorithm to extract the feature and then classification their classes, while the other point is the application; in/out and done the classification with barriers.

In this paper, cross (dense) motion detection is proposed, with and without a barrier (glass barrier because this barrier (wall) is used everywhere), by using a micro-Doppler as motion features. The changing pattern of the reflected signal is studied from radar $2.4 \mathrm{GHz}$ for many experiments scenarios in indoor, outdoor environments and with different light conditions. The combination of KF with $\mathrm{CNN}$ is used as an estimator and classifier; The results show that the proposed work has achieved a detection rate of $98.7 \%$. The enhancement of the present work among the others is provided by making a comparison with other works.

The rest of the paper is organized as follows. In Section 2, the mathematical model of the object's motion and radar signals is presented. The Wall effects; loss and micro-Doppler are explained in Section 3. The proposed algorithms are presented in Section 4. In section 5 a Case study is presented. The simulation and experimental results and discussion of various test case scenarios are presented in Section 6. The conclusions are summarized in Section 7. Finally, future work is presented in section 8 .

\section{The mathematical model of object's motion and radar signals}

The mathematical model of the motion model is shown in Fig. 1 [22]. The displacement $(D)$ function of the moving object is computed as follows;

$$
\begin{array}{r}
D(\mathrm{t})=D_{v} \sin \left(2 \pi f_{v} t\right) \cos \beta_{i} \cos \alpha p \\
\quad(\text { assuming } \alpha=0 \text { and } \beta \mathrm{p}=0)
\end{array}
$$

where;

$D_{v}$ - the displacement in the vertical direction. 


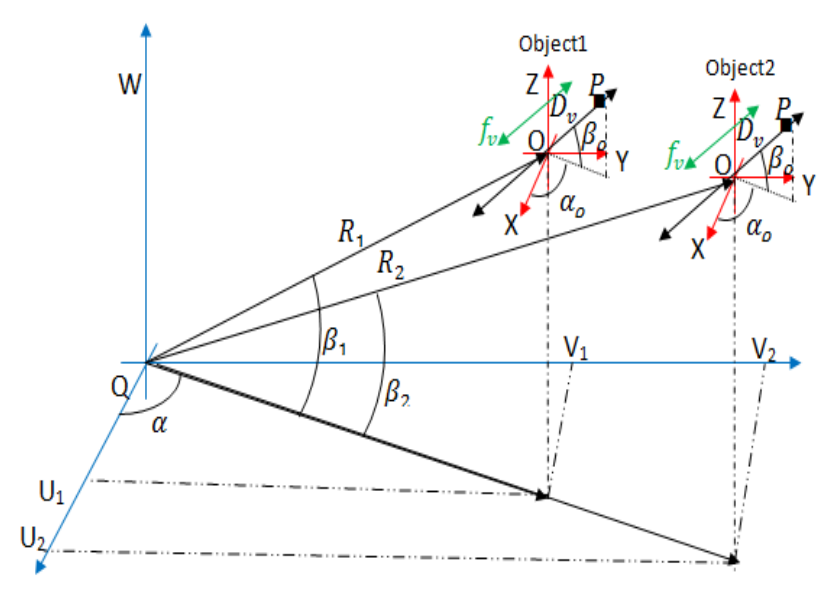

(a)

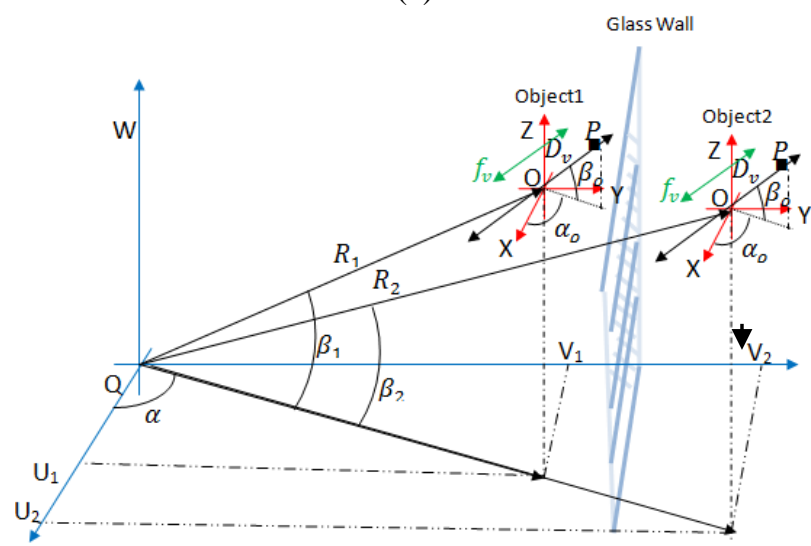

(b)

Figure. 1 Geometry of moving objects concerning the radar location: (a) geometry for radar and two moving objects and (b) geometry for radar and two moving objects with glass wall

The range-time variation according to the target's micro-motion is;

$$
R_{i}(t)=R_{i}+D_{i}(t)
$$

the traveling time of the object which moves at distance $\mathrm{R}$ with velocity $\mathrm{v}$ is;

$$
\tau=2(R+v t) / c_{o}
$$

The radar received signal becomes [23]

$$
\begin{aligned}
& s(\mathrm{t})=\sum_{\mathrm{i}=1}^{\mathrm{n}} \rho_{\mathrm{i}} \mathrm{e}^{\left(\mathrm{j}\left(2 \pi \mathrm{f}_{\mathrm{o}}+\varphi_{\mathrm{i}}(\mathrm{t})\right)\right)} \\
& \varphi_{\mathrm{i}}(\mathrm{t}) \quad=4 \pi \frac{R_{i}(t)}{\lambda} \quad, \mathrm{i}=1,2, . . \mathrm{n}
\end{aligned}
$$

where; fo - carrier frequency.

$\lambda$ - carrier wavelength.

i- object number

$\rho$ - backscattering coefficient = $\pi a^{2} b^{2} c^{2}$

$\overline{\left(\alpha^{2} \sin ^{2} \theta \cos ^{2} \varphi+b^{2} \sin ^{2} \theta \sin ^{2} \varphi+c^{2} \cos ^{2}\right)^{2}}$ $\varphi_{\mathrm{i}}$ - received signal phase.

a,b,c - ellipsoid radii length in the $(\mathrm{x}, \mathrm{y}, \mathrm{z})$ directions respectively, incident aspect angle $(\theta)$ and azimuth angle $(\varphi)$ as [19]. Substituting Eq. (2) in Eq. (5), and then derives its phase from producing the microDoppler as;

$$
f_{m D i}=\frac{W_{v} D_{v}}{\pi \lambda} \cos \beta_{i} \cos \alpha_{\rho} \cos \left(W_{v} t\right)
$$

where;

$\mathrm{W}_{\mathrm{v}}$ - motion frequency in $\mathrm{v}$ direction.

Then, the Range-Doppler (R-D) map is constructed from the matrix Msp, [2];

$$
M s p=N s \times N p
$$

where; $N_{S}-$ Number of sample per each chirp

$$
\begin{aligned}
= & N_{s}=\frac{2 \mathrm{~B} \mathrm{R} \text { max }}{\mathrm{c}} \\
& N_{p}-\text { Number of } \\
= & N_{p}=\frac{\mathrm{c}}{2 \mathrm{f}_{\mathrm{c}} \mathrm{T}_{\mathrm{p}} \mathrm{v}_{\text {res }}} \\
& R_{\text {res }}=\frac{\mathrm{c}}{2 B} \\
& v_{\text {max }}=\frac{\mathrm{c}}{4 \mathrm{f}_{\mathrm{c}} \mathrm{T}_{\mathrm{p}}}
\end{aligned}
$$$$
N_{p}-\text { Number of chirp per measurements }
$$

where;

$$
\begin{aligned}
& v_{r e s} \text { - velocity resolution. } \\
& R_{\text {res }} \text { - range resolution. } \\
& B \quad \text { - bandwidth of transmitting signal. }
\end{aligned}
$$

\section{The wall effects; loss and MD}

The walls affect the penetrating radar signals; these effects appeared on; the dispersion, attenuation, refraction, and reflection, of the return signal and then on the Doppler signatures [9]. These effects, on the signal below $\mathrm{X}$ band, are concentrated in two points; signal phase, and the detectable range, where, the signal-to-noise ratio ( $\mathrm{S} / \mathrm{R})$ of the MD signature is decreased by the wall attenuation [24]. Indeed, when the walls are constructed from the glass or wood or drywall, the attenuation is comparatively small. Consequently, the choice of the frequency for certain application must be precisely selected, because the effect of the material is related to this choice [25]. However, the micro-Doppler modulations of $\mathrm{N}$ structured target's body parts with $\mathrm{Vr}, \mathrm{i}$ as the radial velocity of the $i_{\text {th }}$ structure, the composite micro-Doppler signal has frequency components at $2 \mathrm{~V}_{\mathrm{r}, \mathrm{i}} \mathrm{f}_{\mathrm{o}} / \mathrm{c} \forall \mathrm{i}=1,2, \ldots, \mathrm{N}$, which 


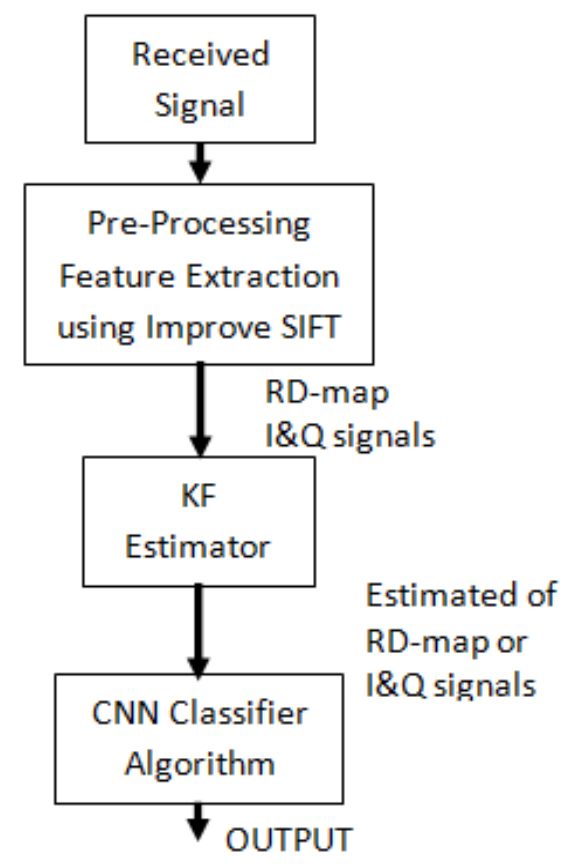

Figure. 2 Proposed an algorithm

are unique to the specific emotional characteristics of that body. Therefore, the Analyzing of the microDoppler signatures for target detection, classification, and recognition is more accurate when analyzed by using the joint time-frequency to provide useful information for that target compatible with its movement with time [26].

\section{Proposed algorithms}

The proposed algorithm is shown in Fig. 2, which is represented by a combination of the KF and $\mathrm{CNN}$. The proposed algorithm concentrates on the received signal. After that, the pre-processing and extracted the features of that signal by using low noise filters (not explained in this work) and improved Stockwell transform respectively. Then estimated the IQ signal or range-Doppler map by KF, after that, the output of estimator filter KF is entered to the CNN classifier to classify this output, to earn more details;

\subsection{Pre-processing (feature extraction)}

Many time-Frequency transforms have been employed by others researches, where that usage is dependent on the required information. Also, the difference between these transforms is the resolution, processing time and the interference cross terms, where each one has advantages on one parameter and disadvantages on the other. The improvement of the Stockwell transform (ST) has been used in this work, which is a hybrid between the short-time
Fourier transform (STFT) and wavelet transform (WT) [27];

$$
\begin{aligned}
& S_{x}^{m, p, k}(\tau, f)= \\
& \int_{-\infty}^{+\infty} x(t) \frac{|f|}{\left(m f^{p}+k\right) \sqrt{2 \pi}} e^{\frac{-(t-\tau)^{2} f^{2}}{2\left(m f^{2}+k\right)^{2}}} e^{-i 2 \pi f t} d t
\end{aligned}
$$

where; $\mathrm{m}, \mathrm{k}$, and $\mathrm{p}$ - Gaussian window control parameters.

\subsection{The estimated Kalman filter model}

The state of moving targets at time $\mathrm{k}$ is a twodimensional vector $\quad X_{k i}=\left(x_{i}, y_{i}, x_{i}^{\prime}, y_{i}^{\prime}\right)$ [28], then the measurement components are;

$$
r_{i}=h\left(X_{k i}\right)+w
$$

Where, w- measurement noise. The target motion model is;

$$
X_{k i+1}=F \cdot X_{k i}+
$$

Where; V- process noise with covariance $\mathrm{Q}$ with acceleration's variances $=10 \frac{\mathrm{m}}{\mathrm{s}^{2}}, \mathrm{~F}$ - transition matrix. The target stat prediction is;

$$
\hat{X}_{k i+1}=F \cdot \hat{X}_{k i}
$$

Predicted error covariance is[29];

$$
P_{k+1 / k}=F P_{k / k} F^{T}
$$

The Kalman filter has estimated the measurements as;

$$
\hat{X}_{k i+1 / k i+1}=\hat{X}_{k i+1 / k i}+K \cdot\left(r_{i} \cdot h\left(X_{k i+1}\right)\right)
$$

The Kalman filter weight is;

$$
K=P_{k+1} H_{k+1}^{T} \mathfrak{C}^{-1}
$$

Where; $\mathfrak{C}-$ innovation matrix $=$ $H_{k+1} P_{k+1 / k} H_{k+1}^{T}+R, R$ - observation covariance noise. Then

$$
P_{k+1 / k+1}=\left(I-K H_{k+1}\right) \cdot P_{k+1 / k}
$$

\subsection{Convolutional neural networks CNN}

Convolutional neural networks CNN represents a powerful tool in a neural network used for classification. The difference between $\mathrm{CNN}$ and 
Table 1. Experimental parameters

\begin{tabular}{|c|c|c|c|}
\hline No, & Parameters & Quantity & Types \\
\hline \multicolumn{4}{|c|}{ Wall } \\
\hline 1 & $\begin{array}{l}\text { Wall } \\
\text { Location }\end{array}$ & 5,20 & Glass, Stone \\
\hline 2 & $\begin{array}{l}\text { Wall } \\
\text { thickness }\end{array}$ & $8 \mathrm{~mm}$ & Glass \\
\hline 3 & \multirow{5}{*}{$\begin{array}{l}\text { Wall } \\
\text { Properties } \\
{[25]}\end{array}$} & 0.00 & Roughness \\
\hline 4 & & $4-9$ & $\begin{array}{l}\text { Dielectric } \\
\text { constant }\end{array}$ \\
\hline 5 & & 0.001 & $\begin{array}{l}\text { Losses } \\
\text { Tahgent }\end{array}$ \\
\hline 6 & & 0.00 & Conductivity \\
\hline 7 & & 2.4 & Permittivity \\
\hline \multicolumn{4}{|c|}{ Moving object } \\
\hline 1 & $\begin{array}{l}\text { Body parts } \\
\text { speed }\end{array}$ & $2-5 \mathrm{~m} / \mathrm{sec}$ & Human 1, 2 \\
\hline 2 & $D v$ & $0.01 \mathrm{~m}$ & \\
\hline 3 & $U$ & $0 \mathrm{~m}$ & \\
\hline 4 & $V$ & $2-5 \mathrm{~m}$ & Human 1,2 \\
\hline 5 & $W$ & $1.66 \mathrm{~m}$ & Human 1,2 \\
\hline 6 & Range & 4,7 & Human 1,2 \\
\hline \multicolumn{4}{|c|}{ Radar } \\
\hline 1 & $\begin{array}{l}\text { Carrier } \\
\text { frequency }\end{array}$ & $2.4 \mathrm{GHz}$ & \multirow{12}{*}{$\begin{array}{l}\text { Radar } \\
\text { Parameters } \\
\text { [33] }\end{array}$} \\
\hline 2 & $\mathrm{BW}$ & $400 \mathrm{MHz}$ & \\
\hline 3 & Sampling & 128 & \\
\hline 4 & $\begin{array}{l}\text { Ant. } \\
\text { Polarization }\end{array}$ & Horizontal/Vertical & \\
\hline 5 & $\begin{array}{l}\text { Range } \\
\text { resolution }\end{array}$ & $37.5 \mathrm{~cm}$ & \\
\hline 6 & $\begin{array}{lr}\begin{array}{l}\text { Number } \\
\text { samples } \\
\text { chirp } N_{s}\end{array} & \text { per } \\
\end{array}$ & 128 & \\
\hline 7 & $\begin{array}{l}\text { Number of } \\
\text { chirps per } \\
\text { measurement } \\
\mathrm{N}_{\mathrm{p}}\end{array}$ & 32 & \\
\hline 7 & R-D map & $2.3 \mathrm{Sec}$ & \\
\hline 8 & Max. Range & $200 \mathrm{~m}$ & \\
\hline 9 & Vres & $0.1 \mathrm{~m} / \mathrm{sec}$ & \\
\hline 10 & $T p$ & $14 \mathrm{msec}$ & \\
\hline 9 & $\begin{array}{l}\text { Sweep Time } \\
\text { FMCW- } \\
\text { Sawtooth }\end{array}$ & $0.5 \mathrm{msec}$ & \\
\hline
\end{tabular}

other conventional neural networks is that; $\mathrm{CNN}$ does not need to pre-extraction of the features. Also, CNN does not need segmentation before neural process beginning, and $\mathrm{CNN}$ is a treatment of the subject which has big data. Despite these the benefits, $\mathrm{CNN}$ is considered more computationally expensive [30]. The CNN structure consists of the input data as a matrix or tensor with a 3D spatial structure, convolution kernel, and output data. The input signal $\mathrm{x}$ is convoluted by filter $\mathrm{f}$ to calculate signal $\mathrm{y}$ as follows [31]:

$$
\begin{gathered}
\mathrm{y}_{\mathrm{i}^{\prime \prime} \mathrm{j}^{\prime \prime} \mathrm{d}^{\prime \prime}}=\mathrm{b}_{\mathrm{d}^{\prime \prime}}+\sum_{\mathrm{i}^{\prime}=1}^{\mathrm{H}^{\prime}} \sum_{\mathrm{j}^{\prime}=1}^{\mathrm{W}^{\prime}} \sum_{\mathrm{d}^{\prime}=1}^{\mathrm{D}} \mathrm{f}_{\mathrm{i}^{\prime} \mathrm{j}^{\prime} \mathrm{d}} \times \\
\mathrm{x}_{\mathrm{i}^{\prime \prime}+\mathrm{i}^{\prime}-1, \mathrm{~d}^{\prime}, \mathrm{d}^{\prime \prime}}
\end{gathered}
$$

where; bd" - neuron offset.

$f_{i j ' d}$ - convolution kernel matrix of the dth $i^{\prime} \times j^{\prime}$.

$\mathrm{x}$ - input data.

$\mathrm{f}$ - convolution filter.

$\mathrm{y}$ - output data

The training data are obtained through forwarding propagation to determine the actual output and then compared with the real data tag competition. To optimize the learnable parameters, a partial derivative of losses with respect learnable parameter is measured, as [30];

$$
w:=w-\alpha \times \frac{\partial L}{\partial w}
$$

where; w -stands for each learnable parameter,

$$
\alpha \text { - stands for a learning rate, and }
$$$$
\mathrm{L} \text { - stands for a loss function. }
$$

\section{Case study}

The experimental parameters used in the practical measurements are shown in Table 1.

Three scenarios were adopted as shown in the Fig. 3, the first one is the detection of two objects with a cross-motion, while the second one is the

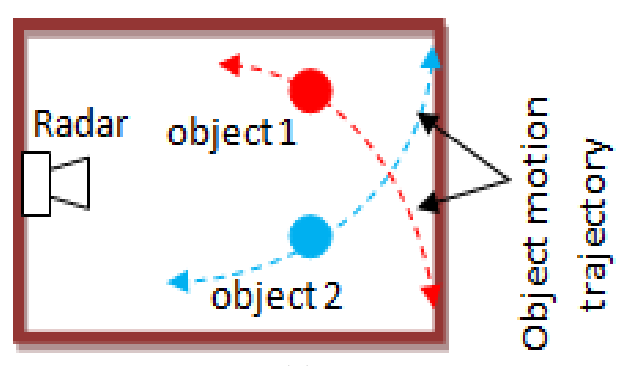

(a)

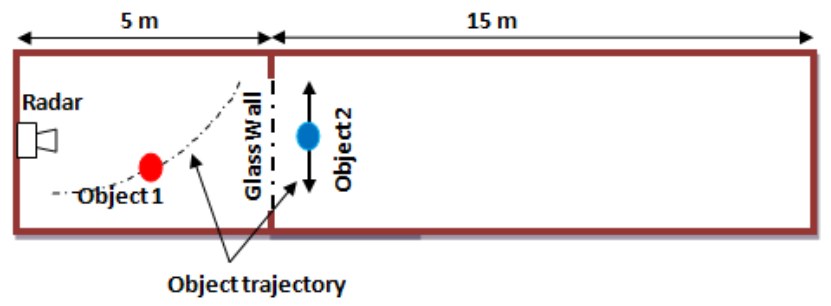

(b)

Figure. 3 Different human motion scenarios: (a) objects motion trajectory without separation and (b) objects motion trajectory with separation by a glass wall without separation 


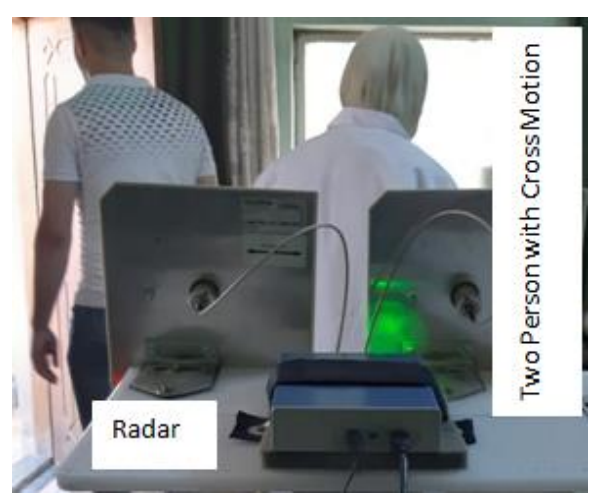

(a)

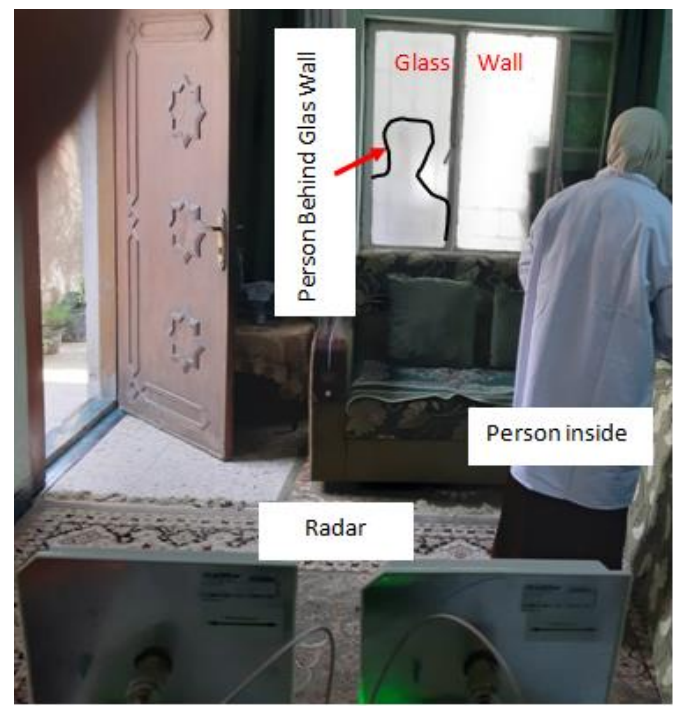

(b)

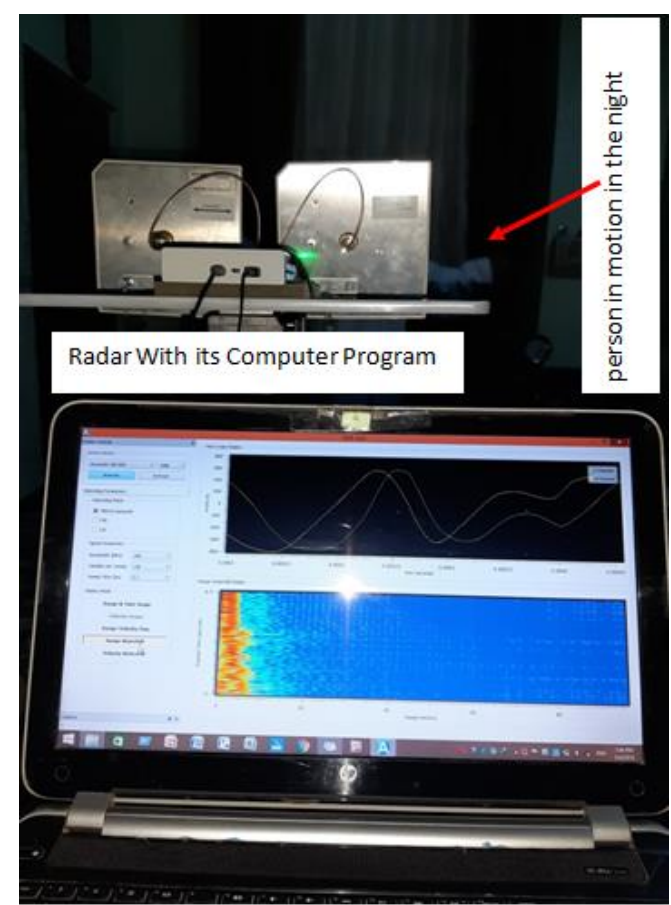

(c)

Figure. 4 Some of the human motion scenarios: (a) crossmotion inside, (b) cross motion with a glass wall, and (c) motion at night same of the first scenario, but with the glass wall between the moving objects. The third scenario is the same as in the first scenario, but the motion is behind the glass wall. The output of the radar is fed to the KF for estimating the signal and then entered to the CNN. The other case study of that combination is to estimate the RD map by $\mathrm{KF}$ and then fed to the CNN. All these motions are done with different light conditions.

To collect the data, the measurements have been carried out on twenty test walking human subjects including different heights and genders with different scenarios. The simulation of the proposed algorithms has been built by using Matlab 2017, while, the practical approach of the proposed algorithm is made by using Ancortek $2.4 \mathrm{GHz}$ radar [33] as in Fig. 4.

\section{Results and discussion}

The result is shown as in Fig. 5 which represents a space, while, the results in Fig. 6 represent the multi rigid object at $5 \mathrm{~m}$ and $18.5 \mathrm{~m}$ away from the radar.

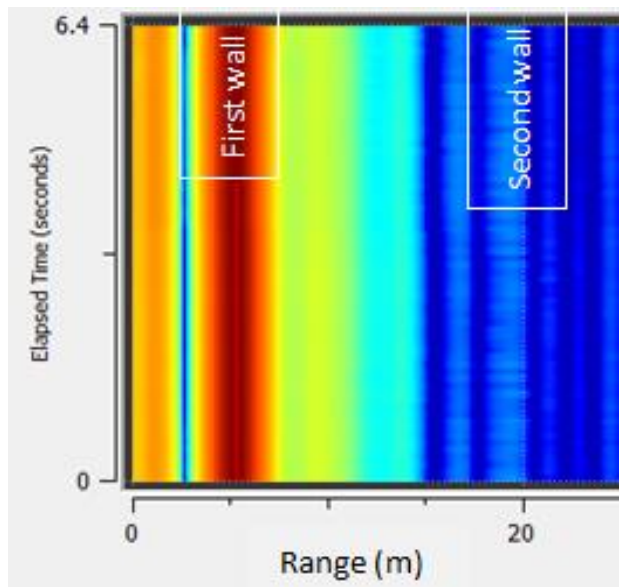

Figure. 5 No subject in space

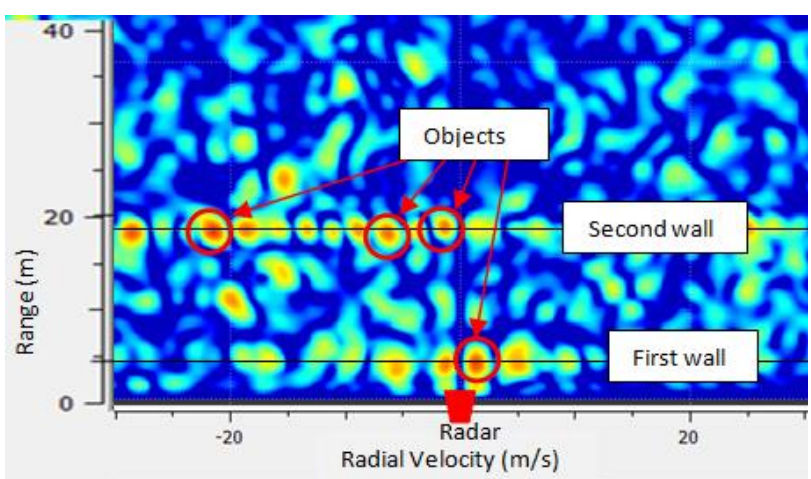

Figure. 6 Moving objects (rigid) at $5 \mathrm{~m}$ and $20 \mathrm{~m}$ 


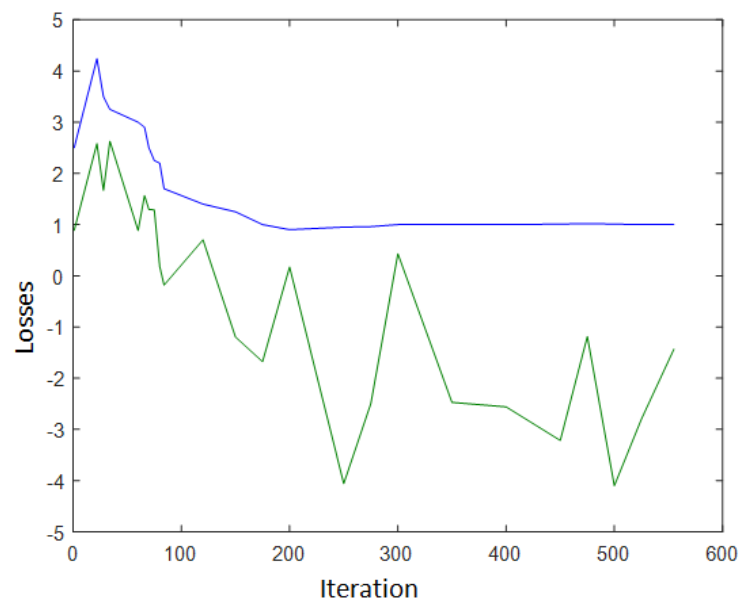

Figure. 7 Three class loss to find the optimal training point
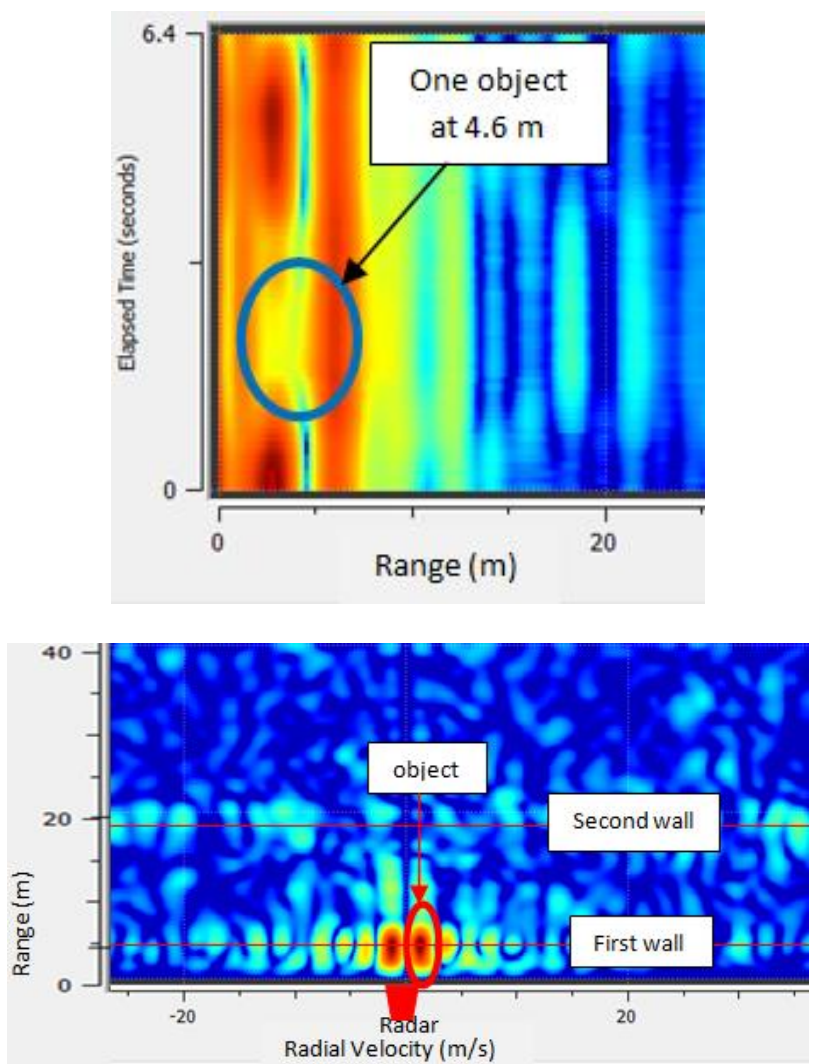

Figure. 8 Fixed the object in $5 \mathrm{~m}$

Therefore, to find the optimal training point of the $\mathrm{CNN}$, the back-propagation using the gradient decent has been used to update the CNN weights by using the partial derivation of cross entropy loss [20], then the bias-variance trade off point is found using validation loss as shown in Fig. 7. Therefore, after 200 iterations, validation loss increases, whereas training continues to decrease. Thus, it can conclude that the model becomes over-fitted beyond 200 iterations, providing the optimal training point. Test data should be applied to this level of iteration to measure model accuracy.

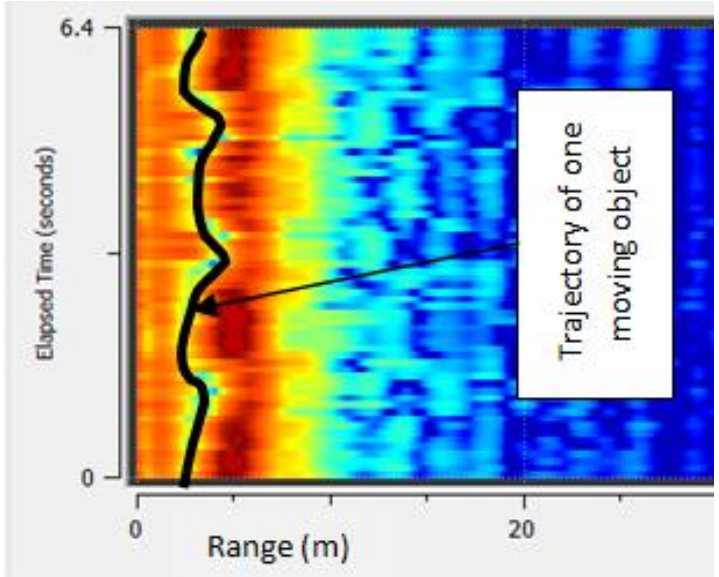

Figure. 9 Human in a zigzag motion (the black line for indication)

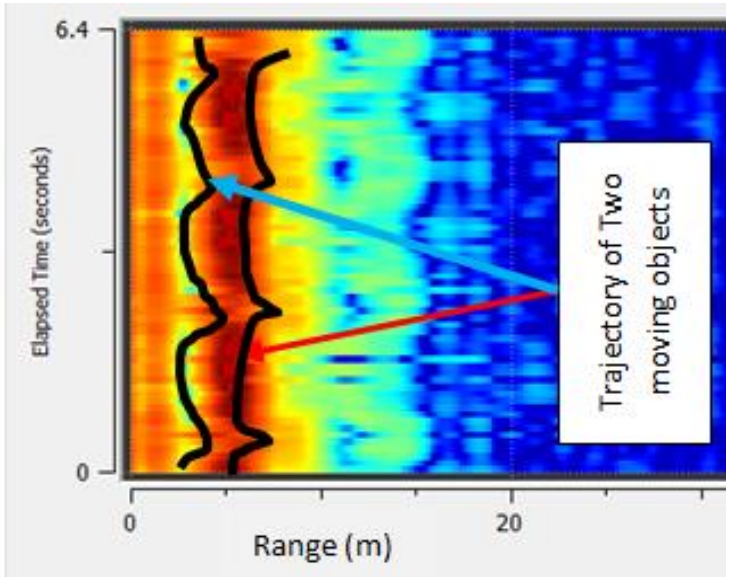

Figure. 10 Two human walking (the black line for indication)

Fig. 8 represents the results for a fixed object at nearly $4.75 \mathrm{~m}$. After the 20 experiments for detection and classification of moving rigid and non-rigid, Fig. 9 presents the detection and classifying of the human in zigzag motion at varying distance from the radar (3-5 m). Fig. 10, represents two humans in a zigzag motion, with varying distances from the radar, where, the first one $(3-5 \mathrm{~m})$ while the other $(5-7 \mathrm{~m})$.

Other experiments were carried out for two humans in motion as shown in Fig. 11. In the first experiment, there are two humans in motion, the first one at $5 \mathrm{~m}$ from the radar while the other at $20 \mathrm{~m}$ from the radar, and the glass wall between them. The effect of the glass wall is clear, where the first human was detected at nearly $5.5 \mathrm{~m}$ and the second was detected at $21 \mathrm{~m}$. Also, at the first time, the proposed algorithm was detecting the first human at $11 \mathrm{~m}$ (error distance caused by the glass wall effect). After that when the Kalman filter is estimated, then it detects the two humans correctly in motion only. 
Table 2. Classification rate of the proposed algorithms

\begin{tabular}{|c|c|c|c|c|c|c|c|}
\hline \multirow{2}{*}{$\begin{array}{l}\text { Proposed } \\
\text { Algorithm }\end{array}$} & \multicolumn{6}{|c|}{ Classification accuracy $\%$ for different motion scenarios } & \multirow{2}{*}{$\begin{array}{l}\text { Overall } \\
\text { Classification } \\
\text { rate } \%\end{array}$} \\
\hline & $\mathrm{NO}$ & OFO & $\mathrm{OMO}$ & TMO & TMOWW & TMOWXW & \\
\hline $\mathrm{KF}+\mathrm{CNN}$ & 100 & 100 & 100 & 99 & 96.7 & 96.5 & 98.7 \\
\hline
\end{tabular}

NO - No object. OFO - One fixed object.

OMO - One moving object. TMO- Two moving objects

TMOWW- Two moving objects with a glass wall.

TMOWXW- Two crosses are moving objects with a glass wall
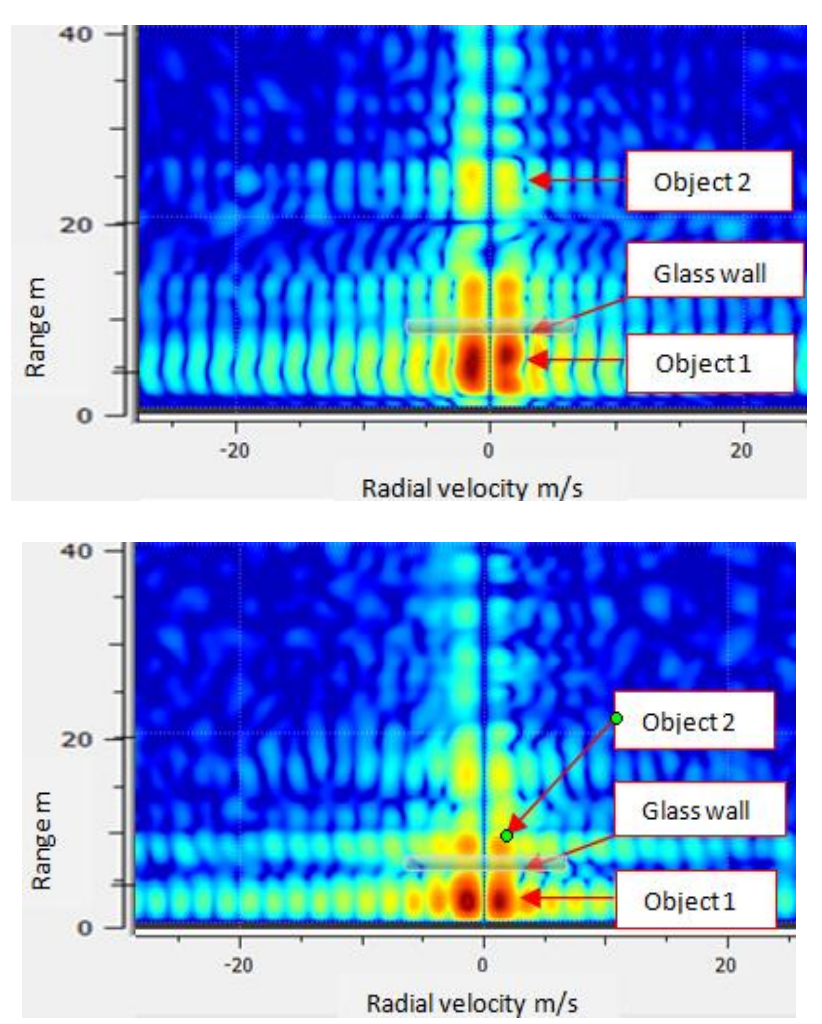

Figure. 11 Two human with a glass wall.

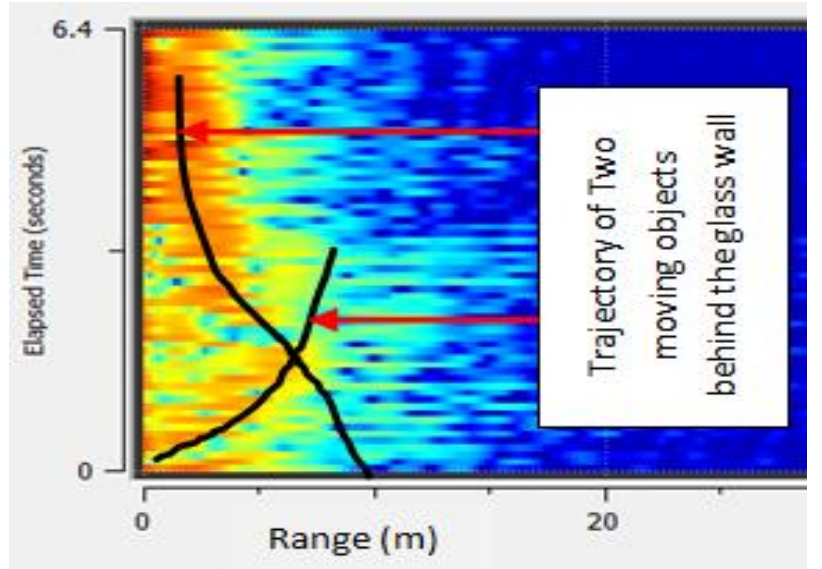

Figure. 12 Two human in motion behind a glass wall

The final experiment was done for two humans in a cross-motion behind the glass wall as shown in Fig. 12 where the two humans move before the glass wall and then exit behind the glass wall by crossmotion as in the same figure.
The detection and classification were made by the proposed combination between the KF and CNN. The KF was used from our previous work [12]. The proposed algorithm was classified the moving objects in two ways; the first one is by entering the IQ signal from the radar receiver to the KF and then to the CNN, while the other is by entering the R-D map to the $\mathrm{KF}$ and then to the $\mathrm{CNN}$. The first way is faster, while the accuracy of the second way is better by a little degree

The summarization of the results for all scenarios and experiments is shown in Table 2. For proving the overperform of the proposed algorithm, two comparisons have been made. The first one for detection and false alarm between the CNN and the used classifier in the compared references as in Table 3. The classifiers in the compared ref.s are Kernel principal components analysis (KPCA), Method of Moments (MoM) enhanced with Fast Multipole Method (FMM), and One-class Support Vector Machine (OSVM). For a fair comparison, the $\mathrm{KF}$ is used with these classifiers as in the proposed work, as shown in Fig. 13, where, the overperform of the CNN is obvious. In this context, the reason for that advancement is because CNN has multiple conventional layers in its core, the output of each prime layer (feature map) is fed as input to the followed layer. This stacked structure enables each layer to learn filters at different levels of the hierarchy. Also, the subsampling layers are resized the feature maps, therefore, the network has seen the data on a different scale, after estimated by KF. Therefore, these stages are working to reduce false alarm. While the use of KPCA is for smoothing and resize the map features. In this context, The MoM is an estimate and simpler in implementation and used with scattering models. Also, OSVM is a non-linear classifier with, related to one class. These traditional algorithms do not reduce the false alarm rate in the detection as well as classification processes. Therefore, the advancement of $\mathrm{CNN}$ with $\mathrm{KF}$ is presented. After that, the number of CNN layers has been tested with different Signal to noise ration and the classification accuracy. Undoubtedly, as the 
Table 3. Comparison between the proposed work and other works

\begin{tabular}{|c|c|c|c|c|c|c|c|}
\hline $\begin{array}{l}\text { Analysis } \\
\text { Parameters } \\
\text { Ref.s \& } \\
\text { Algorithm }\end{array}$ & No. of sensors & $\begin{array}{l}\text { No. of } \\
\text { signals }\end{array}$ & $\begin{array}{l}\text { In/Out } \\
\text { door }\end{array}$ & $\begin{array}{l}\text { With/without } \\
\text { wall }\end{array}$ & $\begin{array}{c}\text { No. of } \\
\text { features }\end{array}$ & $\begin{array}{l}\text { No. of } \\
\text { detected } \\
\text { objects }\end{array}$ & $\begin{array}{c}\text { Classification } \\
\text { rate } \%\end{array}$ \\
\hline [2] Otsu+CNN & $\begin{array}{l}\text { 1/Radar } \\
25 \mathrm{GHz} \\
\end{array}$ & 1 & In & Without & Doppler & Multi & 99 \\
\hline [5] OSVM & $\begin{array}{c}\text { Multi- Wi-Fi } \\
(2.4,5 \mathrm{GHz}) \\
\end{array}$ & Multi & In & Without & 4 & 1 & 90 \\
\hline [9] MoM-FMM & $1 / 2.4 \mathrm{GHz}$ & 1 & In/Out & Without & Doppler & & --- \\
\hline $\begin{array}{c}\text { [19] STFT+KF+ } \\
\text { Tree classifier }\end{array}$ & $1 / 25 \mathrm{GHz}$ & --- & "--- & --- & Doppler & --- & 79 \\
\hline$[20] \mathrm{CNN}$ & 2 & 2 & In/Out & Without & Doppler & 1 & 73.4 \\
\hline [21] CNN & $1 / 25 \mathrm{GHz}$ & 1 & In & Without & Doppler & Multi & $84-98$ \\
\hline $\begin{array}{c}\text { Proposal Worl } \\
\text { KF+CNN }\end{array}$ & $1 / 2.4 \mathrm{GHz}$ & 1 & In/Out & $\begin{array}{l}\text { With and } \\
\text { Without }\end{array}$ & Doppler & $\begin{array}{l}\text { One \& } \\
\text { multi }\end{array}$ & 98.7 \\
\hline
\end{tabular}

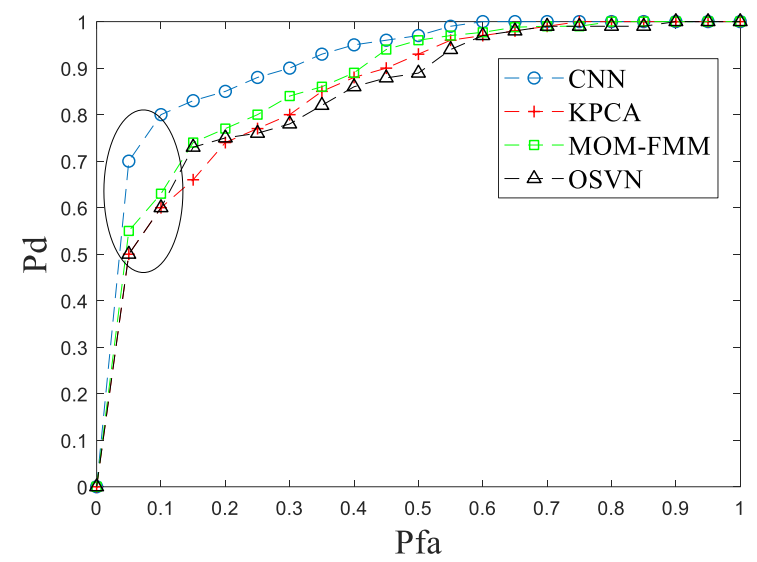

Figure. 13 Probabilities of detection and false alarm of CNN vs KPCA, MoM-FMM \& OSVM

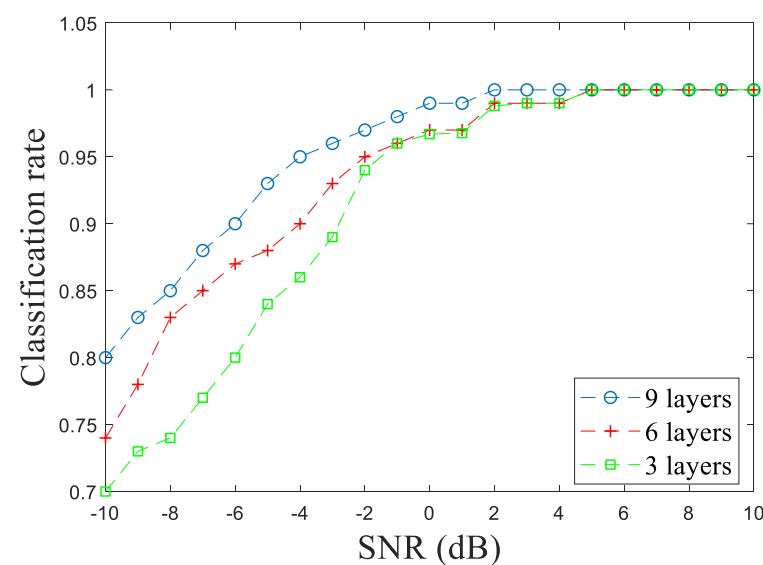

Figure. 14 The classification rate of CNNs with different number of layers versus SNR layer increased the accuracy of classification is increased, but this means the complexity has increased also. Therefore, after many testings, the nine layers have been selected as in Fig. 14.

The proposed model achieves better rejection of the outliers than the KPCA, MoM-FMM, and OSVM, as it is clear in the upper-left-hand corner. This can be explained with the fact that the highly nonlinear transformations in decoder not only provide our model a good reconstructor but also capture features that are relevant to the training data. The other comparison is made with other works as shown in Table 3.

The goal of this work is to improve the classification rate in/out room environments. Therefore, the above results prove that. After that, some nearest work was selected for comparison. The selection is related to the sensor, the feature, the frequency, environmental, the presence or absence of the barrier, the number of detecting objects, and the classification algorithm which were used in these works.

Therefore, from the comparison Table, the advancement of the proposed algorithm is better than another researcher's work. Three references are nearly close to the proposed algorithm, ref $[2,21]$, and [9]. The result of ref [2] is higher than our proposal by $0.3 \%$, but it used $25 \mathrm{GHz}$ radar, and it was working in-room environment and without barrier, while ref [21] was working with the same radar and the environment of ref [2], but with less classification rate than our proposed by $0.7 \%$. Ref 
[9] was working with the same as our work, but without the barrier, and it did not state the classification rate. Therefore, from the summary of the results and the comparisons the proposed algorithms achieve better results. Besides that, our proposal is working with barrier and in/out room environment and with $2.4 \mathrm{GHz}$ radar. This advancement is caused by the robust $\mathrm{CNN}$ and $\mathrm{KF}$ as an individual, and as a combination. Also, the use of the improved of Stockwell transforms has achieved promising results.

\section{Conclusions}

In this paper, human motion detection and classification based on combined the KF with CNN have been proposed as a powerful classifier. Where, after estimated the data by using a Kalman filter, the multi-layers (filters) with sub-sampling layers have been operated to reduce the false alarm and therefore increased the classification rate. In total, twenty different experiments with three scenarios for different (crowded and cross) motion with and without a barrier (glass wall), in/out room environmental have been studied, by using $2.4 \mathrm{GHz}$ radar receiver. The results show that the overperform of the proposed algorithm and the classification accuracy can reach $98.7 \%$. This advancement of the proposed algorithm depends on the efficient Wigner-Ville STFT which is used as a feature extractor or to make RD map. The features are mapped to a 2-D axis as RD map, where it can be observed that most signals in the same categories are distributed in the same cluster. The margins among different clusters are also evident; thus, they are well suited for classification. The results prove that the radar can be represented as robust monitoring sensors in more applications.

\section{Future work}

In the CNN there is an ability to learn each kernel in the convolution layer of different information which is extracted from the received signal. The information includes the phase, frequency, amplitude, and other information can learn to the layers to support the classifier.

\section{References}

[1] T. Boult, "Frame-rate multi-body tracking for surveillance", In: Proc. DARPA Image Understanding Workshop, pp. 305-308, 1998.

[2] S. Abdulatif, Q. Wei, F. Aziz, B. Kleiner, and U. Schneider, "Micro-Doppler Based HumanRobot Classification Using Ensemble and Deep
Learning Approaches", arXiv:1711.09177v3 [cs.CV], 2018.

[3] A. Dewan, T. Caselitz, G. Diego, and T. Burgard, "Motion-based Detection and Tracking in 3D LiDAR Scans", In: Proc. of International Conference on Robotics and Automation, pp. 4508-4513, 2016.

[4] P. Held, D. Steinhauser, A. Kamann, T. Holdgr"un, I. Doric, A. Koch, and T. Brandmeier, "Radar-Based Analysis of Pedestrian Micro-Doppler Signatures Using Motion Capture Sensors", In: Proc. of IEEE Intelligent Vehicles Symposium (IV) Changshu, pp. 787-793, 2018.

[5] J. Liu, L. Wang, J. Fang, L. Uo, B. Lu, and L. Shu, "Multi-Target Intense Human Motion Analysis and Detection Using Channel State Information", Sensors, Vol. 18, No. 3379, 2018.

[6] M. Lahraichi, K. Housni, and S. Mbarki, "Visual Tracking Using Particle Filter Based on Gabor Features", International Journal of Intelligent Engineering and Systems, Vol. 11, No. 4, pp. 147-157, 2018.

[7] A. Krizhevsky, I. Sutskever, and G.Hinton, "Imagenet classification with deep convolutional neural networks", In: NIPS, 2012.

[8] R. Girshick, J. Donahue, T. Darrell, and J. Malik, "Rich feature hierarchies for accurate object detection and semantic segmentation", In: CVPR, 2014.

[9] M. Jose, O. Kilic, V. Dang, Q. Nguyen, and N. Tran, "Analysis of Moving Human MicroDoppler Signature in Forest Environments", Progress in Electromagnetics Research, Vol. 148, pp.1-14, 2014.

[10] D. Orlando and G. Ricci, "Adaptive radar detection and localization of a point-like target", IEEE Trans. Signal Process, Vol. 59, No. 9, pp. 4086-4096, 2011.

[11] A. Aubry, A. De Maio, G. Foglia, C. Hao, and D. Orlando, "Radar detection and range estimation using oversampled data", IEEE Trans. Aerosp. Electron. Syst, Vol. 51, No. 2, pp.1039-1052, 2015.

[12] T. Saeed, M. Al-Muifraje, G. Hatem, J. Abdul Sadah, and A. Abed, "Moving Target Tracking and Classification Based on Micro-Doppler Signatures", International Journal of Intelligent Engineering and Systems, Vol. 12, No. 3, pp. 118-128, 2019.

[13] T. Saeed, M. Al-Muifraje, and G. Hatem, "Through the Wall, Recognize Moving Targets Based on Micro-Doppler Signatures", International Journal of Electrical and 
Computer Engineering, Vol. 8, No. 6, pp. 52275237, 2018.

[14] Y. Kim and T. Moon, "Human detection and activity classification based on micro-doppler signatures using deep convolutional neural networks", IEEE Geoscience and Remote Sensing Letters, Vol. 13, No. 1, pp. 8-12, 2016.

[15] A. Bartsch, F. Fitzek, and R. Rasshofer, "Pedestrian recognition using automotive radar sensors", Advances in Radio Science: ARS, Vol. 10, 2012.

[16] D. Belgiovane and C. C. Chen, "Micro-doppler characteristics of pedestrians and bicycles for automotive radar sensors at $77 \mathrm{ghz}$ ", In: Proc. of 2017 11th European Conference on Antennas and Propagation, pp. 2912-2916, 2017.

[17] S. Abdulatif, F. Aziz, B. Kleiner, and U. Schneider, "Real-time capable micro-doppler signature decomposition of walking human limbs", In: Proc. of 2017 IEEE Radar Conference, 2017.

[18] Y. He, P. Molchanov, T. Sakamoto, P. Aubry, F. L. Chevalier, and A. Yarovoy, "Rangedoppler surface: a tool to analyse human target in ultra-wideband radar", IET Radar, Sonar Navigation, Vol. 9, No. 9, pp. 1240-1250, 2015.

[19] S. Abdulatif, F. Aziz, B. Kleiner, and U. Schneider, "Real-Time Capable Micro-Doppler Signature Decomposition of Walking Human Limbs", arXiv:1711.09175v1 [cs.CV] 25 Nov 2017.

[20] Y. Kwon, S. Shin and S. Kim, "Electroencephalography Based Fusion TwoDimensional (2D)-Convolution Neural Networks (CNN) Model for Emotion Recognition System", Sensors, Vol. 18, No. 1383, 2018.

[21] S. Abdulatif, F. Azizy, K. Armanious, B. Kleiner, B. Yang, and U. Schneider, "Person Identification and Body Mass Index: A Deep Learning-Based Study on Micro-Dopplers", arXiv:1811.07173v2 [cs.CV] 26 Feb, 2019.

[22] C. Clemente, A. Balleri, K. Woodbridge and J. Soraghan, "Developments in target microDoppler signatures analysis: radar imaging, ultrasound and through-the-wall radar", EURASIP Journal on Advances in Signal Processing, Vol. 47, 2013.

[23] R. Radzicki, D. Boutte, V. Taylor, and H. Lee "Standoff CW Radar for Through-the-Wall Detection of Human Heartbeat Signatures", In: Proc. of IEEE Radar Conference, 2016.

[24] S. Sundar R. and A. Majumdar, "Through-Wall Propagation Effects on Doppler-Enhanced
Frontal Radar Images of Humans", In: Proc. of IEEE Radar Conference, 2016.

[25] P. Kumar and T. Kumar, "Ultra-wideband Impulse-based Radar Signals for Through-thewall Imaging", Defence Science Journal, Vol. 62, No. 3, pp. 187-192, 2012.

[26] L. Hachad, F. Mrabti, H. Ghennioui, M. Zouak, "Pilot Decontamination over Time Frequency and Space Domains in Multi-Cell Massive MIMO System", IJECE, Vol. 8, No. 5, part2, 2018.

[27] C. Chen and H. Ling, "Time-Frequency Transforms for Radar Imaging and Signal Analysis", Artech House Radar Library, 2002.

[28] F. Hoffmann, M. Ritchie, F. Fioranelli, A. Charlish, and H. Griffiths, "Micro-Doppler Based Detection and Tracking of UAVs with Multistatic Radar", In: Proc. of IEEE Radar Conference, pp. 1 - 6, 2016.

[29] R. Javier, and Y. Kim, "Application of linear predictive coding for human activity classification based on micro-Doppler signatures", IEEE Geoscience and Remote Sensing Letters, Vol. 11, No. 10, pp. 1831-1834, 2014.

[30] R. Yamashita, M. Nishio, R. Gian Do, and K. Togashi, "Convolutional neural networks: an overview and application in radiology", Insights into Imaging, Vol. 9, pp. 611-629, 2018.

[31] H. Gao, S. Lin, Y. Yang, C. Li, and M. Yang, "Convolution Neural Network Based on TwoDimensional Spectrum for Hyperspectral Image Classification", Hindawi, Journal of Sensors, Vol. 2018, 2018.

[32] A. Moukadem, D. Abdeslam and A. Dieterlen, "Time-Frequency Domain for Segmentation and Classification of Non-stationary Signals", ISTE Ltd and John Wiley \& Sons, Inc, 2014.

[33] ANCORTEK INC, at www.ancortek.com, 2016. 